\title{
Radiofrequency Ablation of the Trochanteric Branches of the Femoral Nerve for the Treatment of Greater Trochanteric Syndrome
}

\author{
Alaa Abd-Elsayed' \\ Joshua M Martens ${ }^{2}$ \\ Kenneth J Fiala ${ }^{2}$ \\ Michael E Schatman $\mathbb{1 D}^{3,4}$ \\ 'Department of Anesthesiology, \\ University of Wisconsin School of \\ Medicine and Public Health, Madison, WI, \\ USA; ${ }^{2}$ University of Wisconsin School of \\ Medicine and Public Health, Madison, WI, \\ USA; ${ }^{3}$ Department of Anesthesiology, \\ Perioperative Care, and Pain Medicine, \\ NYU School of Medicine, New York, NY, \\ USA; ${ }^{4}$ School of Social Work, North \\ Carolina State University, Raleigh, \\ NC, USA
}

Background: Approximately $10-15 \%$ of the population over the age of 60 suffers from hip pain. Greater trochanteric pain syndrome (GTPS) is one of the most common diagnoses in patients with chronic hip pain, includes a number of disorders, and has a broad differential diagnosis. Conservative managements of GTPS, including pharmacologic interventions, physical therapy, chiropractic intervention, acupuncture, and more invasive techniques, such as intra-articular injections, commonly fail to provide patients with sufficient, longterm relief. While radiofrequency ablation (RFA) has been used to treat intra-articular hip pain in the past, there is little evidence for the feasibility of RFA for managing patients with GTPS. This case series builds on previous evidence that cooled radiofrequency ablation (CRF) of the trochanteric branch of the femoral nerve may offer patients with GTPS effective, safe, and lasting pain improvement.

Cases: A series of eight patients with GTPS underwent CRF of the nervus females to the trochanter. CRF procedures were either targeted at the left, right, or bilateral nerves.

Conclusion: This case series provides additional evidence for the safety and effectiveness of CRF of the nervus femoralis to the trochanter for offering long-term pain relief in patients with GTPS. All patients experienced at least two months of relief, with most patients experiencing ongoing relief from the procedure.

Keywords: greater trochanteric pain syndrome, GTPS, radiofrequency ablation, nervus femoralis, trochanter

\section{Introduction}

Approximately $12-15 \%$ of adults over the age of 60 suffer from chronic hip pain, defined as pain most of the days over the past six weeks. ${ }^{1}$ The prevalence of chronic hip pain is even greater among adults who play sports, with estimates ranging from $30-40 \% .^{2,3}$ Not only is this pain debilitating, often impairing an individual's ability to sit and stand, but it often becomes chronic, increasing in incidence with increasing age. ${ }^{4}$ The high prevalence of chronic hip pain might be partially explained by the difficulty clinicians experience in diagnosing the pain. The hip joint is innervated by articular branches of the obturator, femoral, superior gluteal, and sciatic nerves, which complicates the diagnosis of hip pain, as pain is often referred to the hip via these nerves. ${ }^{5,6}$ Patients often present with hip pain accompanied by chronic lumbar or knee pain, or hip pain that is referred from the spine, abdomen, hernial sites, sites of trauma, and several other locations. ${ }^{7,8}$ Greater trochanteric pain syndrome (GTPS) is one of the most common diagnoses in patients with chronic
Correspondence: Alaa Abd-Elsayed Department of Anesthesiology, University of Wisconsin School of Medicine and Public Health, 600 Highland Avenue, B6/ 319 CSC, Madison, WI, 53792-3272, USA $\mathrm{Tel}+$ I 6082636039

Email alaaawny@hotmail.com 
hip pain. GTPS encompasses several disorders of the lateral, peritrochanteric space of the hip, including tears of the gluteus medius and minimus, external coxa saltans tears, and trochanteric bursitis. ${ }^{4}$ Like generalized hip pain, GTPS is relatively common and has a broad differential diagnosis. ${ }^{4,9,10}$ A multicenter observational study conducted by Segal et al estimated the prevalence of unilateral and bilateral GTPS to be $15 \%$ and $8 \%$ in women and $6.6 \%$ and $1.9 \%$ in men ages $50-79$, respectively. ${ }^{11}$ Many patients who present with GTPS will continue to experience chronic pain following both conservative and surgical intervention. ${ }^{4}$ Given the complexity of hip joint pathology, which commonly results in groin and thigh pain, it is often difficult to manage the pain of patients who suffer from GTPS.

Conservative management of GTPS includes acetaminophen, non-steroidal anti-inflammatory drugs (NSAIDs), physical therapy, cooling, chiropractic interventions, and acupuncture. $^{6,12}$ Although conservative measures are effective for some patients, many individuals who suffer from chronic hip pain pursue more invasive measures to manage their pain. Intra-articular injections of local anesthetics and steroid injections can be used in GTPS and have been demonstrated to provide short-term pain improvement in patients with intractable hip pain; however, these interventions often fail to provide long-term benefit to patients. ${ }^{12-14}$ Research investigating potential nervous targets for interventional procedures, such as radiofrequency ablation (RFA), has presented the anterior inferomedial aspect of the hip capsule as the most effective nerve target. ${ }^{15}$ Previous case studies have suggested that percutaneous, continuous radiofrequency lesioning and pulsed radiofrequency (PRF) procedures targeting sensory articular branches within the hip are effective in providing patients with long-term pain relief; however, these procedures come with the risk of motor nerve damage, damage to the femoral neurovascular bundle, neuritis, and neuroma formation. ${ }^{6,18-20}$ Despite these findings, patients with GTPS remain difficult to manage, in part due to the fact that the sensory innervation of the greater trochanter extends from small sensory nerves that branch from the femoral nerve, named as the trochanteric branch of the femoral nerve, and may be spared or incompletely ablated by procedures targeting the hip capsule. ${ }^{12}$ In addition, cadaver dissections have offered additional evidence that no branches of the superior gluteal, inferior gluteal, or sciatic nerve innervate the greater trochanter, presenting the greater trochanter as a region with rather specific innervation in need of direct targeting in order to provide pain relief via interventional procedures. ${ }^{12}$ Much of the available research on the use of RFA to manage patients with hip pain has targeted intra-articular branches of the hip, rather than the trochanteric branch of the nervus femoralis.

Only recently have radiofrequency procedures specifically targeting the femoral nerve and accompanying branches been explored as alternative options for management of patients with GTPS. A case study by Abd-Elsayed et al in 2021 detailed two cases in which cooled radiofrequency ablation (CRF) to the trochanteric branch of the femoral nerve offered pain improvement for over three months in patients with GTPS. ${ }^{21}$ Although the evidence in support of the use of CRF to treat patients with intractable GTPS is growing, there remains a need for further investigation of the best anatomic targets, appropriate patient candidates, and potential negative implications of procedures that target the trochanteric branch of the femoral nerve. This case series describes eight patients with GTPS who underwent CRF of the trochanteric branch of the femoral nerve in order to offer further evidence regarding the effectiveness and safety of the procedure to the growing body of literature on the topic.

\section{Methods}

\section{Patient Selection}

The subjects in this case series were eight consecutive patients who received RFA of the nervus femoralis to the trochanter at the University of Wisconsin Department of Anesthesiology. Patients were given a diagnosis of GTPS if their presenting complaint was consistent with lateral hip pain near the greater trochanter that increased with ambulation, prolonged standing, and other forms of exertion as well as other physical exam findings. Physical exam findings necessary to confirm GTPS were either the presence of a Trendelenburg gait in the absence of antalgic limp with high clinical suspicion or confirmatory testing via FABER test, resisted external derotation test, or tenderness to palpation of the greater trochanter with high clinical suspicion. All patients underwent a trial of conservative management, including pharmacotherapy, physical therapy, and/or steroid injection. Prior to undergoing RFA, patients are generally required to first undergo two separate nerve blocks to confirm that the intended location for RFA will be effective in modulating pain. All patients 
presented in this report have not been previously reported in any other body of work.

\section{Consent and Institutional Review Board Approval}

This case series was deemed exempt by the University of Wisconsin School of Medicine and Public Health Institutional Review Board, as this was a retrospective study with data collection from an Electronic Medical Record (EMR) without the need to contact patients. The Declaration of Helsinki was adhered to throughout the investigation.

\section{Procedure}

The patient is placed in a prone position under standard cardiopulmonary monitoring. The overlying skin is cleaned and draped in a standard, sterile fashion. The femur is viewed under fluoroscopy in the anterior-posterior position in order to confirm placement in a neutral position without excessive rotation. The posterior aspect of the greater trochanter is identified. A line is visualized running from the intersection of the cephalad aspect of the greater trochanter and the femoral neck to the intersection of the caudal aspect of the greater trochanter and the femoral shaft. This line (which we named Al's posterior trochanteric line) is divided into thirds, with a target at the intersection of the proximal and middle thirds and another at the intersection of the middle and distal thirds. This line is intended to correspond with the trochanteric branch of the femoral nerve. In order to avoid nearby insertions of the gluteus medius and quadratus femoris muscles, the posterior aspect of the greater trochanter is targeted.

The patient receives 0.5 to $1 \mathrm{cc}$ of $1 \%$ lidocaine at the intended entry site of RFA. Fluoroscopic guidance is utilized to identify targets and advance via 22-gauge, 3.5-5inch spinal needles, which will be used for nerve blocks, to the periosteum. Once the needles are in place, Omnipaque $^{\circledR}$ (iohexol) $240 \mathrm{mgI} / \mathrm{mL}$ contrast is injected to confirm a soft-tissue contrast pattern covering the posterior aspect of the greater trochanter. A negative aspiration must be confirmed in order to proceed with injection of $1.5 \mathrm{~mL}$ of $0.25 \%$ bupivacaine at each site. Needles are withdrawn. Nerve blocks are considered successful if 50\% pain relief over several hours is achieved following two separate blocks.

Following successful nerve blocks, a 22-gauge, $10 \mathrm{~cm}$ RFK cannula with $10 \mathrm{~mm}$ active tip is placed following

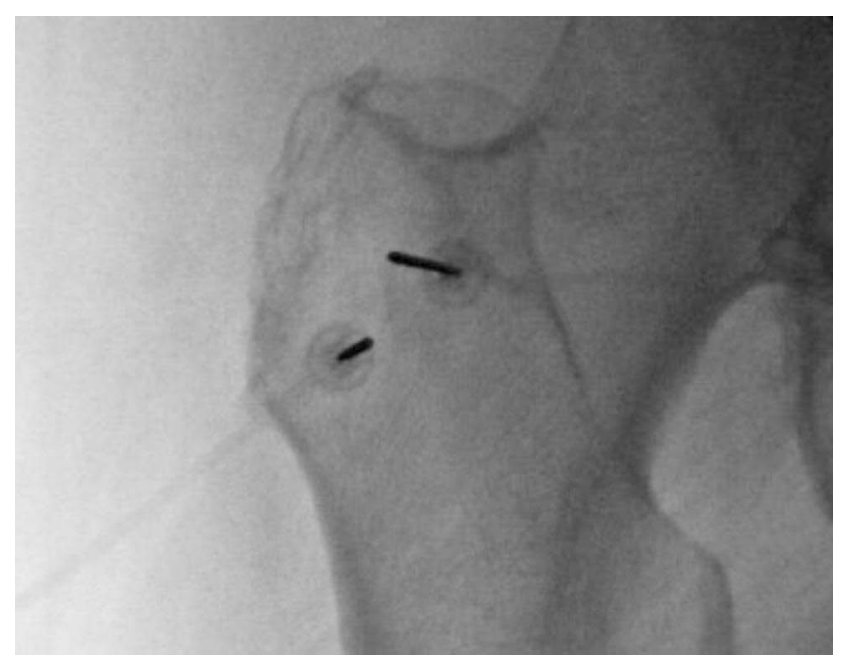

Figure I Depicts a posterior view of radiofrequency probe at the left greater trochanter.

the same approach under fluoroscopic guidance as the confirmatory nerve blocks. The stylet is removed and the RF probe is inserted through the needle (Figure 1), followed by motor testing to confirm no stimulation of the distal lower extremity. Two percent lidocaine is injected prior to initiating cooled RFA, consisting of $80^{\circ} \mathrm{C}$ for 165 seconds utilizing lesion mode. The probes and trochars are removed following ablation.

\section{Follow-up}

Patient follow-up was completed under guidelines for onemonth and three-month follow-up appointments. During these follow-up visits, patient side effects were discussed, potential damage to motor function was assessed via physical exam, and postprocedure pain scores were obtained using a visual analogue scale (VAS).

\section{Results \\ Case I}

This was a 30-year old female patient with a history of trochanteric bursitis of the right hip who presented to the interventional pain clinic with chronic low back pain that initially began following a cesarean-section in 2016 and was exacerbated by a motor vehicle accident (MVA) in 2018. Following the MVA, the patient reported exacerbated sharp, achy, chronic bilateral axial low back pain and new right hip pain. Upon initial presentation, the patient's back pain was rated at a $8 / 10$ on visual analogue scale (VAS), a 3.5/10 at its best and a 10/10 at its worst. The patient reported minimal improvement with trigger 
point injections (TPI), ibuprofen, heating cream, and one scoop daily of ortho molecular's collagen powder. The patient underwent one greater trochanteric bursa injection, one lumbar epidural steroid injection at L5-S1, and two diagnostic right nervus femoralis trochanteric branch nerve blocks prior to undergoing RFA intervention.

The patient underwent right nervus femoralis to the trochanter nerve RFA under fluoroscopic guidance on October 28, 2020. 16 days following the procedure, the patient's right trochanteric pain improved from $8 / 10$ prior to the procedure to less than $1 / 10$ postprocedure, reporting a $95 \%$ improvement. The patient underwent one additional lumbar epidural steroid injection following the procedure; however, this was targeted at her axial low back pain, which persisted following RFA intervention. Overall, the patient reported an improvement in quality of life following RFA. The patient continues to report ongoing pain relief from the procedure.

\section{Case 2}

This was a 48-year old female patient with a history of fibromyalgia and trochanteric bursitis of both hips who presented to the interventional pain clinic with a complaint of bilateral hip pain secondary to a diagnosis of greater trochanteric bursitis. Her pain was rated at a 7/10 on VAS. She underwent previous trigger point injections (TPI) and two bursa injections, which provided over $80 \%$ relief of her pain. She reported minimal improvement with duloxetine, gabapentin, etodolac, physical therapy, psychotherapy, steroid injections, and nerve blocks.

The patient underwent right nervus femoralis to the trochanter nerve RFA under fluoroscopic guidance on January 20, 2021. Forty-seven days after the procedure, the patient reported $75 \%$ improvement of her pain. One hundred and sixty-two days postprocedure, following a left trochanteric nerve RFA, the patient continued to report $80 \%$ improvement in her pain in both hips. The patient reported no negative effects from the procedure; however, she did continue to receive follow-up and treatment for other pain syndromes, such as chest wall pain status postmastectomy and chronic migraine headaches.

Approximately five months following the initial rightsided RFA procedure, the patient underwent left nervous femoralis to the trochanter nerve RFA under fluoroscopic guidance on May 5, 2021. Fifty-seven days after her leftsided RFA and 162 days after her right-sided RFA, the patient continued to report $80 \%$ improvement in her pain in both hips. The patient continued her medications and also began a course of oral antibiotics to treat a new bladder infection; however, she reported no other negative effects from RFA and reported significant improvement in her quality of life. The patient continues to report ongoing pain relief from the procedure.

\section{Case 3}

This was a 59-year-old female patient with a history of greater trochanteric pain syndrome who presented to the interventional pain clinic with a complaint of bilateral low back and right hip pain. She rated her hip pain as $6 / 10$ on VAS and described the pain as a nagging and constant. She noticed minimal relief with physical therapy, chiropractic intervention, acupuncture, ibuprofen, gabapentin, topical lidocaine, and left greater trochanteric bursa injection.

The patient underwent right nervous femoralis to the trochanter nerve RFA under fluoroscopic guidance on February 10, 2021. Thirty-three days after the procedure, the patient reported a $45 \%$ reduction of her pain. The patient did note some initial increases of the pain in her right hip, which may have been attributable to pain at the site of the procedure, although the patient further pointed to her back and gluteal region when describing the site of the transient pain increase. Seventy-six days postprocedure, the patient reported that her pain had returned to the baseline levels she was experiencing prior to the procedure.

\section{Case 4}

This was a 57-year old female patient with a history of arthritis who presented to the interventional pain clinic with a complaint of low back, left lateral thigh pain, and right leg pain. She rated the pain in her thighs as a $5 / 10$ on VAS, noting that the pain was $3 / 10$ at its best and $10 / 10$ at its worst. The patient noticed minimal improvement with chiropractic interventions, physical therapy, spinal traction, medial branch blocks, lumbar transforaminal epidural injections, and TPI. She also reported minimal improvement with gabapentin, amitriptyline, hydrocodone, and IV lidocaine infusions for pain. She previously had a spinal cord stimulator (SCS) implanted for back pain and an infusion pump, which offered minimal relief.

The patient underwent bilateral nervous femoralis nerve RFA under fluoroscopic guidance on February 17, 2021. Ninety-seven days after the procedure, the patient's pain remained improved $85 \%$. The patient continues to report ongoing relief from the procedure. 


\section{Case 5}

This was a 64-year-old female patient with a history of greater trochanteric bursitis who presented to the interventional pain clinic with a complaint of multisite pain. She rated her pain as a $8 / 10$ on VAS, noting the pain was $4 / 10$ at best and 10/10 at its worst. The patient reported minimal improvement with baclofen, duloxetine, neurontin, nabumetone, diclofenac gel, physical therapy, TENS, steroid injections, TPIs, and TAP blocks. Prior to the procedure, the patient was managed with a implanted infusion pump.

The patient underwent bilateral nervous femoralis to the trochanter nerve RFA under fluoroscopic guidance on March 31, 2021. Thirty-five days post-procedure, the patient's pain had improved to a $6 / 10$, reaching $9 / 10$ at its worst. One hundred and sixty-nine days after the procedure, the patient reported a $40 \%$ improvement in pain. The patient continues to report ongoing relief from the procedure.

\section{Case 6}

This was a 54-year-old female patient with a history of trochanteric bursitis who presented to the interventional pain clinic with a complaint of low back, hip, and right buttock pain following spinal fusion for scoliosis and a labral tear over 10 years prior to her RFA procedure. She did not begin to notice significant pain until two years prior to the RFA procedure. She rated her pain as $8 / 10$ on. The patient noted minimal improvement with physical therapy, chiropractic interventions, steroid injections, TENS, and pharmacotherapy.

The patient underwent right nervous femoralis to the trochanter nerve RFA under fluoroscopic guidance on March 31, 2021. Forty days postprocedure, her pain improved to $3 / 10$ on VAS, reaching $8 / 10$ at its worst. Her pain continued to reduce with standing and walking, and continued to worsen with sitting and lying on her right side. The patient continues to see ongoing pain relief from the procedure.

\section{Case 7}

This was a 52-year-old female patient with a history of left trochanteric bursitis who presented to the interventional pain clinic with a complaint of left-sided low back pain and headaches. The patient rated her pain as a $5 / 10$. The patient reported minimal improvement with physical therapy, pharmacotherapy, and left trochanteric bursa injection.
The patient underwent left nervous femoralis to the trochanter nerve RFA under fluoroscopic guidance on April 21, 2021. Thirty-four days postprocedure, her pain had improved to $0 / 10$ on VAS. The patient did note that excessive activity would bring on the pain, but found significant improvements to her quality of life following the procedure. The patient continued to take hydrocodone, ibuprofen, and naproxen following the procedure.

\section{Case 8}

This was a 64-year female old patient who presented to the interventional pain clinic with a complaint of low back and right leg pain. She rated her pain $7 / 10$, noting that the pain is $5 / 10$ at best and $8 / 10$ at worst. The patient noticed minimal improvement with physical therapy, chiropractic intervention, TENS unit, steroid injections, and pharmacotherapy. The patient noted an injury 38 days prior to her RFA after falling in the snow, exacerbating her hip and back pain.

The patient underwent bilateral nervous femoralis to the trochanter nerve RFA under fluoroscopic guidance on June 7, 2021. Thirty-nine days postprocedure, her pain had improved to $2 / 10$ on VAS. The patient also reported improvement of her quality of life, noting the ability to lie on both sides and sleep through the night following the procedure. The patient continues to report ongoing pain relief from the procedure.

\section{Summary of Results}

This case series examined eight of our patients who underwent right, left, or bilateral nervous femoralis to the trochanter nerve RFA in order to modulate chronic hip and leg pain. Three patients underwent right nervous femoralis to the trochanter RFA only, one underwent left-sided RFA, and three underwent bilateral RFA. There was a single patient who underwent both right and left nervous femoralis to the trochanter nerve RFA, with each procedure separated by approximately five months. The average age of the patients was 48 and all patients were female. All patients reported a reduction in pain following the procedure, with an average pain reduction of $71.4 \%$. The average preprocedural pain score was 6.9 and the average postprocedural pain score was 2.1 . The median preprocedural pain score was 7 and the median postprocedural pain score was 2 . The average follow-up for pain improvement occurred at 53.7 days postprocedure. Table 1 depicts a summarization of patient demographic data, pain scores, and average values, standard deviations, and rangesas for 
Table I Patient Demographics and Pain Scores Pre- and Post-cRFA

\begin{tabular}{|c|c|c|c|c|c|c|c|c|}
\hline Case & Age & Sex & BMI & $\begin{array}{l}\text { Laterality } \\
\text { of } \\
\text { Procedure }\end{array}$ & $\begin{array}{l}\text { Preprocedure Pain } \\
\text { Score (VAS) - Worst }\end{array}$ & $\begin{array}{l}\text { Postprocedure Pain } \\
\text { Score (VAS) - Worst }\end{array}$ & $\begin{array}{l}\text { \% Reduction } \\
\text { in Pain Score }\end{array}$ & $\begin{array}{l}\text { Duration of } \\
\text { Improvement }\end{array}$ \\
\hline 1 & 30 & $\mathrm{~F}$ & 44.6 & Right & 8 & 1 & $87.5 \%$ & Ongoing \\
\hline 2 & 48 & $F$ & 30.8 & Right, Left & 7 & 1.4 & $80 \%$ & Ongoing \\
\hline 3 & 59 & $F$ & 37.1 & Right & 6 & 3.3 & $45 \%$ & 76 days \\
\hline 4 & 57 & $F$ & 22.2 & Bilateral & 6.5 & 0.975 & $85 \%$ & Ongoing \\
\hline 5 & 64 & $F$ & 33.5 & Bilateral & 8 & 4.8 & $40 \%$ & Ongoing \\
\hline 6 & 54 & $\mathrm{~F}$ & 20.7 & Right & 8 & 3 & $62.5 \%$ & Ongoing \\
\hline 7 & 52 & $\mathrm{~F}$ & 31.8 & Left & 5 & 0 & $100 \%$ & Ongoing \\
\hline 8 & 64 & $F$ & 29.1 & Bilateral & 7 & 2 & $71 \%$ & Ongoing \\
\hline Average & 48 & - & 31.2 & - & 6.9 & 2.1 & $71.4 \%$ & - \\
\hline $\begin{array}{l}\text { Standard } \\
\text { deviation }\end{array}$ & 9.7 & - & 6.8 & - & 0.96 & 1.4 & $19.4 \%$ & - \\
\hline Range & $30-64$ & - & $\begin{array}{c}20.7- \\
44.6\end{array}$ & - & $5-8$ & $0-4.8$ & $40-100 \%$ & - \\
\hline
\end{tabular}

all analyzed data. A paired $t$-test comparing preprocedure and postprocedure pain scores yielded a $p$-value less than 0.0001. All patients saw improvement in pain at their initial follow-up; however, the ability to assess duration of improvement was limited in that seven of eight patients continued to see ongoing improvement at the time of writing of this case series. The single patient who experienced only transient relief experienced 76 days of improvement prior to returning to baseline. In addition, all of the procedures performed in this case series were performed within one year of the writing of this case series report, which limited the extent to which long-term pain relief could be assessed.

\section{Discussion}

The results of this case study provide additional evidence, supplementing previous work by Abd-Elsayed et al, for the effectiveness of nervus femoralis to the trochanter nerve CRF for the management of patients with intractable pain from GTPS. All patients in the series saw improvement in pain following CRF and the reduction in pain, presented as differences between pre- and postprocedure pain scores, was statistically significant at a significance level $\leq 0.05$. Though the analysis of duration of improvement was limited by the fact that all but one of the patients continued to see ongoing improvement in pain at the time of publication, the ongoing nature of the pain relief provided to most patients provides additional evidence for the effectiveness of the intervention. This case series also provides additional insight into the risks associated with the procedure that prior research was unable to assess given limited sample size. While this case series was limited to eight patients, one of the patients did experience a paradoxical increase in pain following intervention, which is the first documented side effect in patients undergoing this procedure.

Case 3 discusses a patient who experienced a transient increase in right-sided hip pain immediately following the procedure. It is possible that the patient experienced pain at the site of ablation secondary to excess tissue damage or trauma sustained during needle placement and thermal coagulation. Of note, this patient also suffered from low back pain and did point to her black and gluteal regions when describing the new pain that she experienced following RFA. There remains the potential that the new, transient pain that she experienced reflected referred pain from her back. Despite this case of increased back pain status post RFA, the results of this case series do not suggest that patients with concomitant low back pain should not be considered for nervous femoralis to the trochanter RFA. To the contrary, seven of the eight patients within the series suffered from chronic low back pain, all of whom 
reported substantial relief of their pain from the procedure, many of whom experienced significant, lasting improvement that contributed to an improvement in patient quality of life. Other side effects reported following the procedure were minimal and likely unrelated to the procedure. One patient did begin additional pain management following the procedure for unrelated pain secondary to a bladder infection.

The mechanism of pain relief offered by radiofrequency ablation remains poorly understood. There is evidence to suggest that thermal coagulation induced by the radiowaves denatures the proteins of the peripheral nerve, thus preventing nociceptive conduction along it. ${ }^{12}$ Additional research by Letcher and Goldring suggests that there is a discrepancy in the extent of damage caused by heat on myelinated and unmyelinated nerve fibers, which may further explain the specificity of RFA procedures; however, provided that the ganglion of the nerve remains intact, the nerve will have the capacity to regenerate and begin sending nociceptive signals once this occurs. ${ }^{20}$ Ablation of the branch of the nervus femoralis to the trochanter, which was conducted in this review, is likely to provide patients with intractable GTPS relief due to the fact that the small sensory branches of this nerve are often the only innervation to the greater trochanter. There remain outstanding questions regarding the most effective method of RFA to provide patients with intractable pain related to GTPS with lasting relief, as there is limited literature on other RFA targets for patients with GTPS and no randomized control studies comparing CRF and other RFA modalities have been conducted to date. Future clinical studies should investigate differences in effectiveness, safety, and long-term outcomes of these procedures.

The results of this case series provide additional evidence for the safety of CRF procedures targeting the trochanteric branch of the nervus femoralis in patients with GTPS. No lasting negative outcomes were observed in any patients who underwent the procedure and no novel symptoms related to the procedure appeared in the weeks and months following intervention. In addition, this case series provides additional evidence for the long-term efficacy of CRF in managing patients with intractable pain related to GTPS. While some patients did not experience relief from their first procedure, there is evidence that some patients who see limited benefit from an initial RFA might have additional, longer-lasting relief following a second procedure. ${ }^{21}$ The variability of the effect of RFA in subsequent procedures remains an area in need of further investigation. This case series included a single patient who underwent two RFA procedures, separated by five months, and saw lasting relief from both; however, these procedures were on different sides of the hip and the results are likely more comparable to that of a single, bilateral RFA of the nervous femoralis to the trochanter than two subsequent RFA procedures to the same side. Future clinical studies should investigate the efficacy of subsequent RFA procedures following an initial RFA that, at a minimum, provided the patient with minimal, but noticeable relief.

\section{Conclusion}

This case series described eight patients who underwent left, right, or bilateral nervous femoralis to the trochanter CRF to modulate their chronic, intractable hip pain related to GTPS. The results of this case series provide additional evidence to the growing body of literature for the safety of the procedure, as well as the effectiveness of the procedure in improving patient's pain for lasting periods of time. The patients reviewed in this case series saw statistically significant improvements in pain following the procedure, lasting at least two months, and reported minimal side effects. There was a single case in which a patient reported a subjective increase of chronic low back pain following the procedure, which likely impacted the relief the patient experienced and should be investigated in future clinical studies. Larger clinical trials should be conducted to further evaluate the long-term side effects and effectiveness of the procedure, as well as the impact on additional RFA procedures on the same nerve in providing relief to patients.

\section{Disclosure}

Dr Michael E Schatman is a research consultant for Modoscript, outside the submitted work. Dr. AbdElsayed is a consultant of Medtronic, Avanos, and Averitas. The authors report no other conflicts of interest in this work.

\section{References}

1. Christmas C, Crespo CJ, Franckowiak SC, Bathon JM, Bartlett SJ, Andersen RE. How common is hip pain among older adults? Results from the Third National Health and Nutrition Examination Survey.. The Journal of family practice. 2002;51(4):

2. Thorborg K, Rathleff MS, Petersen P, Branci S, Hölmich P. Prevalence and severity of hip and groin pain in sub-elite male football: a crosssectional cohort study of 695 players. Scandinavian journal of medicine \& science in sports. 2017;27(1). doi:10.1111/sms.12623

3. Langhout R, Weir A, Litjes W, et al. Hip and groin injury is the most common non-time-loss injury in female amateur football. Knee surgery, sports traumatology, arthroscopy: official journal of the ESSKA. 2019;27(10). doi:10.1007/s00167-018-4996-1 
4. Ahuja V, Thapa D, Patial S, Chander A, Ahuja A. Chronic hip pain in adults: current knowledge and future prospective. J Anaesthesiol Clin Pharmacol. 2020;36(4):450. doi:10.4103/joacp.joacp_170_19

5. Birnbaum K, Prescher A, Hepler S, Heller K-D. The sensory innervation of the hip joint - an anatomical study. Surg Radiol Anat. 1998;19(6):371-375. doi:10.1007/s00276-997-0371-5

6. Wu H, Groner J. Pulsed radiofrequency treatment of articular branches of the obturator and femoral nerves for management of hip joint pain. Pain Pract. 2007;7(4):341-344. doi:10.1111/j.15332500.2007.00151.x

7. Wilson JJ, Furukawa M. Evaluation of the patient with hip pain. Am Fam Physician. 2014;89(1):27-34.

8. Strauss E, Nho S, Kelly B. Greater trochanteric pain syndrome. Sports Med Arthrosc Rev. 2010;18(2):113-119. doi:10.1097/ JSA.0b013e3181e 0b2ff

9. Tibor LM, Sekiya JK. Differential diagnosis of pain around the hip joint. Arthroscopy. 2008;24(12):1407-1421. doi:10.1016/j. arthro.2008.06.019

10. Williams BS, Cohen SP. Greater trochanteric pain syndrome: a review of anatomy, diagnosis and treatment. Anesth Analg. 2009;108(5):1662-1670. doi:10.1213/ane.0b013e31819d6562

11. Segal NA, Felson DT, Torner JC, et al. Greater Trochanteric Pain Syndrome: Epidemiology and Associated Factors. Archives of Physical Medicine and Rehabilitation. 2007;88(8). doi:10.1016/j. apmr.2007.04.014

12. Genth B, von Düring M, von Engelhardt LV, Ludwig J, Teske W, von Schulze-Pellengahr C. Genth B, von Düring M, von Engelhardt LV, Ludwig J, Teske W, von Schulze-Pellengahr C. Clinical anatomy (New York, NY). 2012;25(8). doi:10.1002/ca.22035

13. Flanagan J, Casale FF, Thomas TL, Desai KB. Intra-articular injection for pain relief in patients awaiting hip replacement. Ann $R$ Coll Surg Engl. 1988;70(3):156-157.
14. Heywang-Köbrunner SH, Amaya B, Okoniewski M, Pickuth D, Spielmann RP. CT-guided obturator nerve block for diagnosis and treatment of painful conditions of the hip. Eur Radiol. 2001;11 (6): 1047-1053. doi:10.1007/s003300000682

15. Tomlinson J, Ondruschka B, Prietzel T, Zwirner J, Hammer N. A systematic review and meta-analysis of the hip capsule innervation and its clinical implications.. Scientific Reports. 2021;11(1):10471053. doi: $10.1038 / \mathrm{s} 41598-021-84345-\mathrm{z}$

16. Saberski L, Fitzgerald J, Ahmad M. Cryoneurolysis and radiofrequency lesioning. Pract Manage Pain. 1998;3:1007-1106.

17. Kawaguchi M, Hashizume K, Iwata T, Furuya H. Percutaneous radiofrequency lesioning of sensory branches of the obturator and femoral nerves for the treatment of hip joint pain. Reg Anesth Pain Med. 2001;26(6):576-581. doi:10.1097/00115550-200111000-00016

18. Bhatia A, Hoydonckx Y, Peng P, Cohen SP. Radiofrequency Procedures to Relieve Chronic Hip Pain. Regional Anesthesia and Pain Medicine. 2018;43(1):72-83. doi:10.1097/AAP.0000000000000694

19. Bhatia A, Hoydonckx Y, Peng P, Cohen S. Radiofrequency procedures to relieve chronic hip pain. Reg Anesth Pain Med. 2018;43 (1):72-83. doi:10.1097/AAP.0000000000000694

20. Letcher FS, Goldring S. The effect of radiofrequency current and heat on peripheral nerve action potential in the cat. J Neurosurg. 1968;29 (1):42-47. doi:10.3171/jns.1968.29.1.0042

21. Lord SM, Barnsley L, Wallis BJ, McDonald GJ, Bogduk N. Percutaneous radio-frequency neurotomy for chronic cervical zygapophyseal-joint pain. New Engl J Med. 1996;335(23):1721-1726. doi:10.1056/nejm199612053352302
Journal of Pain Research

\section{Publish your work in this journal}

The Journal of Pain Research is an international, peer reviewed, open access, online journal that welcomes laboratory and clinical findings in the fields of pain research and the prevention and management of pain. Original research, reviews, symposium reports, hypothesis formation and commentaries are all considered for publication. The manuscript

\section{Dovepress}

management system is completely online and includes a very quick and fair peer-review system, which is all easy to use. Visit http:// www.dovepress.com/testimonials.php to read real quotes from published authors. 